PRULIFLOXACIN VERSUS LEVOFLOXACIN IN THE TREATMENT OF CHRONIC BACTERIAL PROSTATITIS: A PROSPECTIVE, RANDOMIZED, DOUBLE-BLIND TRIAL

Giannarini G. ${ }^{1}$, Mogorovich A. ${ }^{1}$, Valent F. ${ }^{2}$, Morelli G. ${ }^{1}$, De Maria M. ${ }^{1}$, Manassero F. ${ }^{1}$, Barbone F. ${ }^{2}$, Selli C. ${ }^{1}$

${ }^{1}$ University of Pisa, Urology, Pisa, Italy, ${ }^{2}$ University of Udine, Epidemiology, Udine, Italy

Introduction \& Objectives: Oral fluoroquinolones represent the first choice of treatment for patients with documented chronic bacterial prostatitis (CBP), and the recommended duration is at least 4 weeks. Since the various available drugs present different pharmacokinetic and pharmacodynamic properties and different microbial resistance profiles, the aim of the present study is to compare the efficacy and safety of Prulifloxacin, a new broad-spectrum oral fluoroquinolone with long half-life and high urinary concentrations, with those of Levofloxacin in the treatment of men with CBP.

Material \& Methods: A total of 96 consecutive patients with a clinical diagnosis of CBP and current laboratory evidence of infection were randomized to receive a 4-week oral course of either Prulifloxacin $600 \mathrm{mg}$ or Levofloxacin $500 \mathrm{mg}$ once daily. All subjects were evaluated with the Meares-Stamey test and the validated Italian version of the National Institutes of Health Chronic Prostatitis Symptom Index (NIH-CPSI) at baseline and one week after therapy completion. Patients with microbiological eradication were evaluated for recurrent infection with the Meares-Stamey test 6 months after therapy completion. The primary end point was microbiologic eradication. The secondary end points were clinical efficacy, assessed by reduction in the NIH-CPSI, recurrent infection rate at 6 months, and safety.

Results: The microbiologic eradication rate was $72.73 \%$ for Prulifloxacin and $71.11 \%$ for Levofloxacin $(p=0.86)$. The clinical success rate was not significantly different, since the reduction in the NIH-CPSI score from study entry to study completion was 10.75 (from 17.22 to 6.47 ) for Prulifloxacin and 10.73 (from 17.33 to 6.6) for Levofloxacin ( $p=0.98$ ). After 2 weeks of treatment there was a trend toward a greater reduction of the symptom score for Prulifloxacin (5.29) than for Levofloxacin (4.80) $(p=0.1005)$. At 6 months a lower proportion of patients on Prulifloxacin $(n=5)$ had a positive Meares-Stamey test compared to Levofloxacin $(n=11)$, the difference being only marginally significant $(p=0.1008)$. Safety was comparable in the two groups, with a rate of treatment-related adverse events of $18.18 \%$ for Prulifloxacin and $22.22 \%$ for Levofloxacin $(p=0.79)$.

Conclusions: The present results suggest that Prulifloxacin $600 \mathrm{mg}$ orally once daily is at least as effective and safe as Levofloxacin $500 \mathrm{mg}$ orally once daily administered for 4 weeks in the treatment of men with CBP. Therapy with both drugs enables the great majority of patients to achieve microbiological eradication of the infecting strain and to significantly improve the symptom complex. Recent studies have shown that the active metabolite of Prulifloxacin has a greater tendency than the other fluoroquinolones to penetrate within both bacteria and phagocytic cells, and this is reflected by a trend to an earlier resolution of subjective symptoms.

\section{PHYSICAL ACTIVITY FOR MEN WITH CHRONIC PROSTATITIS CHRONIC PELVIC PAIN SYNDROME NOT SATISFIED WITH CONVENTIONAL TREATMENTS: COULD IT REPRESENT A VALID OPTION? THE PHYSICAL ACTIVITY AND MALE PELVIC PAIN TRIAL : A DOUBLE BLIND, RANDOMIZED STUDY}

Giubilei G. ${ }^{1}$, Mondaini ${ }^{2}{ }^{2}$, Minervini A. ${ }^{1}$, Nelli F. ${ }^{1}$, Saieva C. $^{3}$, Lanzi F. ${ }^{1}$, Lapini A. ${ }^{1}$ Serni S. ${ }^{1}$, Bartoletti R. ${ }^{2}$, Raugei A. ${ }^{1}$, Carini M. ${ }^{1}$

${ }^{1}$ Department of Urology, University of Florence, Careggi Hospital, Florence, Italy., Urology, Florence, Italy, ${ }^{2}$ Department of Urology, Santa Maria Annunziata Hospital, Florence, Italy., Urology, Florence, Italy, ${ }^{3}$ Molecular and Nutritional Epidemiology Unit, Center for Cancer Prevention, Scientific Institute of Tuscany, Florence, Italy

Introduction \& Objectives: Chronic prostatitis/chronic pelvic pain syndrome is a major healthcare burden. Affected patient quality of life is poor and currently no investigated treatments have significant long-term benefit. We performed a preliminary investigation of the role of physical activity and its effects on select patients with chronic prostatitis/ chronic pelvic pain syndrome.

Material \& Methods: Between 2002 and 2004 we recruited a volunteer sample of 231 eligible males 20 to 50 years old with chronic prostatitis/chronic pelvic pain syndrome who were unresponsive to conventional treatments and free of any contraindication for moderate intensity physical exercise. This group was screened and, if in accordance with study inclusion/exclusion criteria, patients were randomized into 2 groups. Partecipants were randomly assigned to the aerobic exercise group (52) and the placebo/stretching and motion exercises group (51). Main outcome measures were the Italian version of the National Institute of Health Chronic Prostatitis Symptom Index, Beck Depression Inventory, State Anxiety Inventory- $Y$ and a pain intensity visual analog scale administered at baseline, and 6 and 18 weeks.

Results: At 18 weeks 36 subjects $(75 \%)$ in the aerobic exercise group vs $40(81.63 \%)$ in the placebo/stretching and motion exercises group completed the 18-week program and evaluation. Differences between the 2 groups were found in total National Institutes of Health Chronic Prostatitis Symptom Index, pain and quality life impact subscales, and pain visual analog score (ANCOVA $p=0.006,0.0009,0.02$ and 0.003 respectively).

Conclusions: Improvements in the aerobic exercise group were significantly superior compared to those in the placebo/ stretching and motion exercises group. Aerobic exercise represents a valid treatment option and it should be further investigated in a larger study with longer followup.

\section{ARTERIAL HEMODYNAMIC OF THE PROSTATE AND NEUROPHYSIOLOGY OF PELVIC FLOOR AT PATIENTS WITH THE CPICPPS III AND EFFICIENCY ANALGESIC PREPARATIONS}

Kogan M. ${ }^{1}$, Belousov.$^{1}$, Shangichev A. ${ }^{1}$, Palenyj A. ${ }^{2}$, Afoko P. ${ }^{1}$

${ }^{1}$ Rostov State Medical University, Urology, Rostov on Don, Russia, ${ }^{2}$ Rostov State Medical University, Diagnostic Radiology, Rostov on Don, Russia

Introduction \& Objectives: The contemporary investigations of the pathogenesis of Chronic Pelvic Pain Syndrome (CPPS) have shown that in the inflammatory (IIIA) CPPS the basis of the pain syndrome is inflammation, whilst in the non-inflammatory (IIIB) CPPS vascular and neurological factors are responsible. The primary intent of the CP/CPPS III B therapy is correction the pain syndrome.

Material \& Methods: We've investigated the intraorganic prostate hemodynamic (the density of bloodstream and the top indices of the arterial blood flow's speed), the neurophysiology of the pelvic floor's muscles and compared the efficacy specimen with different mechanism of action in the pain syndrome's therapy, which have patients with CP/CPPS III B. 65 patients with diagnosis CP/CPPS III B and pain quantification in Visual Analog Scale (VAS) 4-8 have been analyzed. There were 30 patients in the group with the form III A; 35 patients in the group with the form III $B$. In the control group were 13 healthy volunteers. All patients were $20-40$ years old. 32 patients with CP/CPPS III B had therapy for 12 weeks: 12 patients took Flupirtin $100 \mathrm{mg} \times 4$ times per day; 10 patients took Celecoxib $200 \mathrm{mg} x$ one time per day. The effects of the drugs were assessed at 1,4 and 8 weeks of therapy using the VAS and compared with placebo (10 patients).

Results: Hemodynamic parameters in the group IIIA were statistically doubtful $(p>0.05)$ and smaller than in the control, but reduced in all quantifications. Patients with IIIB in $88.6 \%$ of cases had hemodynamic parameters, which were smaller than more $50 \%$ in control $(p<0.01)$. At the same time the amplitude of the potential of the motor unit (PMU) was increased in $58.3 \%$ of cases $(1087,2 \pm 534,6 \mu \mathrm{V})$ and high-amplitude PMU $(>1000 \mu \mathrm{V})$ was recorded in $50 \%$ of cases. The increase the bulbocavernous reflex $>41 \mathrm{~m} / \mathrm{sec}$ was recorded in $58.3 \%$ of cases and reduction cortical somato-sensory potentials that were generated by stimulating the pudendal nerve in $75 \%$ of cases. The bigger analgesic effect was reached in the group of patients with Flupirtin. Intensity of pain was reduced by 8 week at $67.2 \%$ in placebo group, in the group with Celecoxib reduction was $43.7 \%$.

Conclusions: The reduction of blood flow's speed against a background reduction of bloodstream's density by CP/CPPS IIIB indicate organ ischemia Our investigations revealed sub-clinical pathology in patients with CPPS IIIB in $66.7 \%$ cases. Among these $75.0 \%$ are seen to have peripheral neuropathy and $41.7 \%$ have pudendal axonopathy. Analgesic effect of both pharmacologic preparations was observed already by the end of the first week and continued to rise right up to the eighth week of investigation. Flupirtin had a better clinical effect compared to Celecoxib.

\section{ESTIMATION OF STANDARD THERAPY CHRONIC PELVIC PAIN SYNDROM (CP/CPPS III) AT MEN}

Kogan M. ${ }^{1}$, Shangichev A. ${ }^{1}$, Mikashinovich Z. ${ }^{2}$, Chernogubova E. ${ }^{3}$, Shimunov G. ${ }^{2}$, Belousov.$^{1}$, Afoko A. ${ }^{1}$

${ }^{1}$ Rostov State Medical University, Urology, Rostov on Don, Russia, ${ }^{2}$ Rostov State Medical University, Biochemistry, Rostov on Don, Russia, ${ }^{3}$ Southern Russian Research Centre of the Russian Acadamy of Science, Urology, Rostov on Don, Russia

Introduction \& Objectives: The Activation of kallikrein-kinin system (KKS) in the blood is a biochemical marker of intensity of inflammatory process in the prostate. The nitric oxide (NO) stars in the mechanisms of development of inflammation. The purpose of the research is to develop criterion of monitoring efficiency of treatment inflammatory and aninflammatory forms CP/CPPS.

Material \& Methods: Some parameters KKS of bloods and NO- systems in dynamic of standard pharmacotherapy CP/CPPS III at 21 patients with form CP/ CPPS IIIA and at 25 patients with form CP/CPPS IIIB before treatment and in 1 month after its beginning were investigated. The treatment of patients with form IIIA was ofloxin $400 \mathrm{mg} 2$ times per day, doxazosin $2 \mathrm{mg} 1$ time per day and warm baths. With form IIIB was doxazosin $2 \mathrm{mg} 1$ time per day, diclofenac $25 \mathrm{mg} 3$ times per day and massage of the prostate. All participants of research were randomize by double blind method in groups with the standard therapy CP/CPPS III and with the placebo.

Results: The reception of placebo did not influence on investigated indexes. After the treatment at patients with CP/CPPS IIIA the proteolytic potential of blood was enlarged by $18,7 \%(p<0.05)$, contents $\mathrm{NO}$ has decreased on $30,8 \%(p<0.001)$, has reached the control level. At patients with CP/CPPS IIIB after the treatment, activity kallikrein has increased on 50,8 \% ( $<<0.001)$, activity of $\alpha 2$-macroglobulin has enlarged by $20,6 \%(p<0.05)$ and contents NO has not changed.

Conclusions: The activity of kallikrein of bloods and production of NO in macrophages at CP/CPPS are biochemical markers of the efficiency treatment. Disbalance KKS in the dynamic of treatment CP/CPPS testifies to its inefficiency, that demands development the other therapy of the form IIIB. 